\title{
Atomic-scale ETEM Observation of Chemical Reactions on the Surface of Nanomaterials
}

Hideto Yoshida

Osaka University, Ibaraki, Osaka, Japan

Chemical reactions on the surface of materials are important for catalyst, synthesis of nanomaterials, and nanodevices. Environmental transmission electron microscopy (ETEM) is one of the most powerful experimental methods for revealing atomic structures and dynamics of the surface of nanomaterials during chemical reactions [1]. However, it is necessary to pay attention to the effects of electron irradiation on the observed phenomena.

We have investigated atomic structures and dynamics of nanomaterials under reaction conditions by ETEM while taking into account the effects of electron irradiation [2-7]. We have found that the surface of $\mathrm{Pt}$ nanoparticles is oxidized in $\mathrm{O}_{2}$ gas under electron irradiation (Figure 1). The surface of $\mathrm{Pt}$ nanoparticle was not oxidized in $\mathrm{O}_{2}$ gas at low electron current densities $\left(0.1 \mathrm{~A} / \mathrm{cm}^{2}\right)$. Upon increasing the current density from $0.1 \mathrm{~A} / \mathrm{cm}^{2}$ to $4.0 \mathrm{~A} / \mathrm{cm}^{2}$, the surface structure changed to the Pt oxides [2]. It has also been observed that the reduced Co nanoparticles on $\mathrm{Nb}_{2} \mathrm{O}_{5}$ are oxidized even in a vacuum with the aid of electron irradiation [3]. In addition, we have found an unexpected gas-solid reaction that occurs in an electrically-biased Pd nanogap with $\mathrm{N}_{2}$ gas under electron irradiation (Figure 2). A PdN compound was synthesized on the surface of the positive Pd electrode at room temperature [6]. It is most likely that the above mentioned oxidation of $\mathrm{Pt}$ and Co nanoparticles and the formation of PdN compounds were induced by electronic excitations of metal atoms and gas molecules, involving high-energy electrons and associated secondary electrons that are emitted from the samples by the irradiation of high-energy electrons.

We have developed phase-locked TEM [8]. In phase-locked TEM, the specimen is irradiated by the phaselocked strobe electron beam whose pulse width, interval, and intensity are controllable. Using phaselocked TEM combined with a fast CMOS camera, we can investigate the effect of electron irradiation on the observed phenomena quantitatively by TEM. We will show the preliminary results of applying phaselocked TEM to the study of catalyst nanoparticles.

This work was supported in part by SENTAN, JST, Grant-in-Aid for scientific Research (A), Grant No. 25246003, Grant No.16H02074 and "Dynamic Alliance for Open Innovation Bridging Human, Environment and Materials" from the Ministry of Education, Culture, Sports, Science and Technology of Japan (MEXT).
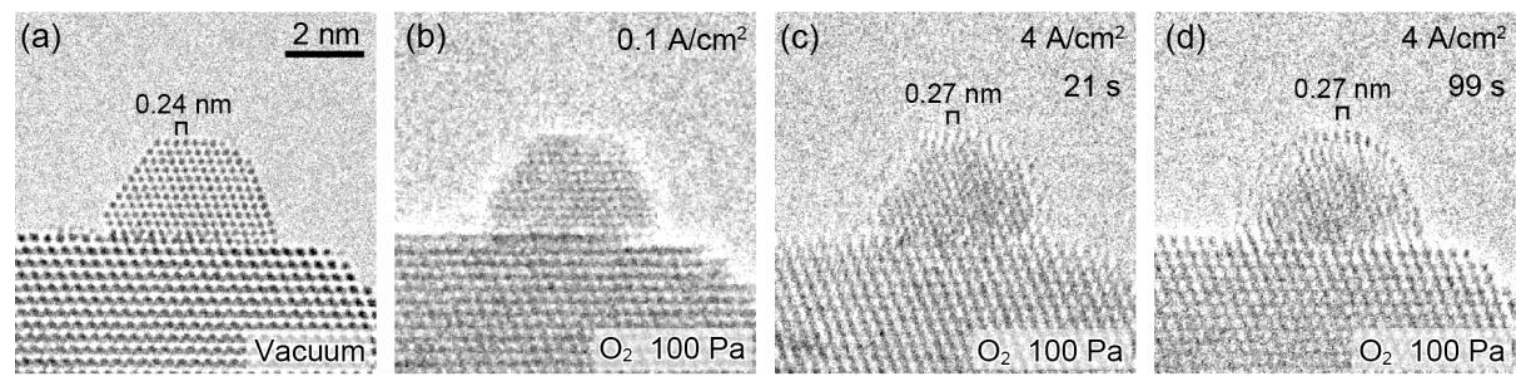
Figure 1. Oxidation of a $\mathrm{Pt}$ nanoparticle in $\mathrm{O}_{2}$ gas under electron irradiation [2]. ETEM images of the $\mathrm{Pt}$ nanoparticle (a) in a vacuum, (b) in $100 \mathrm{~Pa} \mathrm{O}_{2}$ gas at electron current densities of $0.1 \mathrm{~A} / \mathrm{cm}^{2}$, and (c), (d) in $100 \mathrm{~Pa} \mathrm{O}_{2}$ gas at electron current densities of $4.0 \mathrm{~A} / \mathrm{cm}^{2}$. Times shown in (c) and (d) are elapsed times after the current density was changed from $0.1 \mathrm{~A} / \mathrm{cm}^{2}$ to $4.0 \mathrm{~A} / \mathrm{cm}^{2}$. The spacing of lattice fringes in (c), (d) was estimated to be $0.27 \mathrm{~nm}$. The spacing can be explained by Pt oxides.

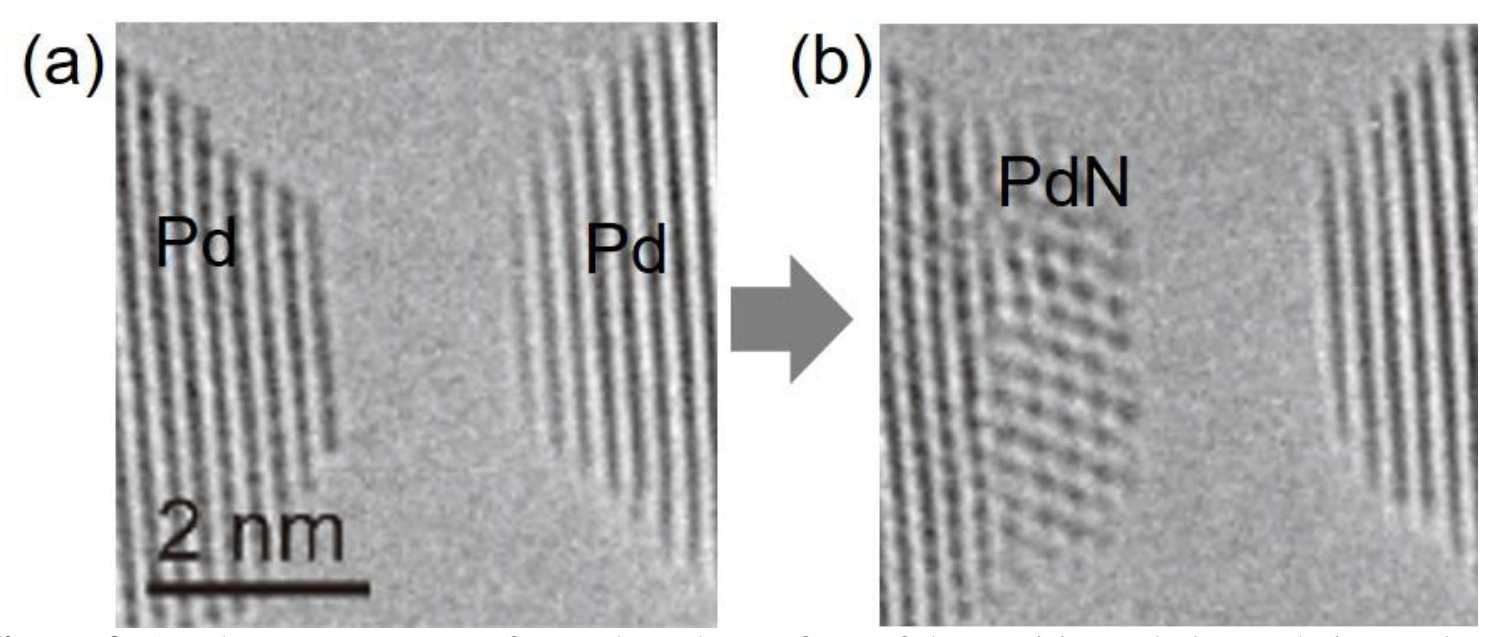

Figure 2. A PdN nanostructure formed on the surface of the positive Pd electrode in an electrically-biased nanogap with $\mathrm{N}_{2}$ gas [6]. The biasing voltage between the electrodes, is (a) $0 \mathrm{~V}$ and (b) $8.0 \mathrm{~V}$.

\section{References}

[1] S. Takeda, Y. Kuwauchi, and H. Yoshida, Ultramicroscopy 151 (2015) 178.

[2] H. Yoshida, H. Omote, and S. Takeda, Nanoscale 6 (2014) 13113.

[3] J. H. den Otter, H. Yoshida, C. Ledesma, D. Chen, and K. P. de Jong, J. Catal. 340 (2016) 270.

[4] H. Yoshida, Y. Tomita, K. Soma, and S. Takeda, Nanotechnology 28 (2017) 195301.

[5] N. Kamiuchi, K. Sun, R. Aso, M. Tane, T. Tamaoka, H. Yoshida, and S. Takeda, Nat. Commun. 9 (2018) 2060.

[6] T. Tamaoka, R. Aso, H. Yoshida, and S. Takeda, Nanoscale 11 (2019) 8715.

[7] R. Aso, Y. Ogawa, T. Tamaoka, H. Yoshida, and S. Takeda, Angew. Chem. Int. Ed. 58 (2019) 16028.

[8] K. Soma, S. Konings, R. Aso, N. Kamiuchi, G. Kobayashi, H. Yoshida, and S. Takeda, Ultramicroscopy 181 (2017) 27. 